\title{
Stem Water Content for Crape Myrtle in Response to Drought, Cold, and Disease Stress
}

\author{
Chao Gao $\mathbb{D}^{1,2}$ Yue Zhao $\mathbb{D}^{3,4}$ and Yandong Zhao $\mathbb{D}^{3,4}$ \\ ${ }^{1}$ School of Computer and Information Engineering, Beijing Technology and Business University, Beijing 100048, China \\ ${ }^{2}$ Beijing Key Laboratory of Big Data Technology for Food Safety, Beijing Technology and Business University, Beijing 100048, China \\ ${ }^{3}$ School of Technology, Beijing Forestry University, Beijing 100083, China \\ ${ }^{4}$ Beijing Laboratory of Urban and Rural Ecological Environment, Beijing Municipal Education Commission, Beijing 100083, China
}

Correspondence should be addressed to Chao Gao; gaochao9158@sina.com

Received 27 October 2019; Revised 28 December 2019; Accepted 8 January 2020; Published 7 February 2020

Guest Editor: Zhenxing Zhang

Copyright (c) 2020 Chao Gao et al. This is an open access article distributed under the Creative Commons Attribution License, which permits unrestricted use, distribution, and reproduction in any medium, provided the original work is properly cited.

\begin{abstract}
The abiotic and biotic stresses including drought, cold, and disease stress are linked by the fact that they all decrease the availability of water to plant cells. In previous studies, some physiological factors related with plant water status, such as stem sap flow, leaf transpiration rate, and water potential, were used to assess the effects of these stresses on plants. But there are few studies about the effects of these stresses on stem water content (StWC) which can be measured by a novel SWR sensor. In this study, crape myrtle was selected as an experimental subject and its StWC was observed in four experiments including no stress, drought, cold, and disease stress. Before conducting stress experiments, the StWC and environmental and physiological parameters were synchronously monitored under unstressed conditions on a typical day in summer. In the experiment of drought stress, the StWC was monitored under different gradients of soil moisture. In the experiment of cold stress, the StWC was monitored in warm and cold weather, respectively. In the experiment of disease stress, the StWC was monitored under different frequencies of disease treatment. The results showed that the correlation coefficients between StWC and PAR and VPD were larger than 0.5 and the correlation coefficients between StWC and Pn, Tr, Gs, and Ci were larger than 0.8 under no stress. The diurnal mean of StWC decreased firstly, then remained stable for a period of time, and eventually continued to fall under drought stress. On the whole, there was a negative correlation between the diurnal mean of StWC and the degree of drought stress. The StWC showed opposite diurnal variation rules in warm and cold weather. There was a positive correlation between the diurnal range of StWC and the degree of cold stress. The diurnal minimum, maximum, and mean of StWC showed a positive correlation with the health level of plants and the diurnal range of StWC showed a negative correlation with the health level of plants. In conclusion, the StWC can be used as a qualitative evaluation index of the degree of the three types of stress.
\end{abstract}

\section{Introduction}

Plants often suffer from continuous exposure to various abiotic and biotic stresses in natural and agricultural settings. The stresses, such as drought, cold, and disease stress are serious threats to plants and result in the deterioration of the environment. Hence, it is important to distinguish whether plants are under a certain stress.

Drought stress usually occurs when available water in soil is reduced and meteorological conditions cause continuous loss of water by evaporation or transpiration [1]. Drought stress can be quantified as a decrease in water potential $\left(\psi_{\mathrm{w}}\right)$
[2]. In most cases, the first response of plants for drought stress is to avoid low $\psi_{\mathrm{w}}$ by adjusting stomatal conductance such that the rates of water loss and water uptake remain balanced [3]. As the drought stress becomes severe, the plant is no longer able to keep a balance between water loss and uptake. Under this condition, some additional mechanisms, such as solutes accumulation $[4,5]$ and cell wall hardening [6] play an important role in keeping plants from dehydration. As the drought stress becomes more severe, it is increasingly difficult for the plant to avoid dehydration. In this case, some additional mechanisms, including protective proteins [7], metabolic changes [8], and reactive oxygen 
species (ROS) detoxification [9] become important for plants in enduring dehydration. In the process of continuous dehydration, plant tissues and organs may suffer from damages, indicating that the plant need to be irrigated immediately.

Cold stress usually can be classified into two types, chilling stress and freezing stress [10]. Compared with chilling stress, freezing stress is more harmful to plants. From the macro perspective, water begins to freeze in the large vessels of the xylem in leaves and stems where dilute sap has a higher freezing point than other solutions in plant [11]. From the micro perspective, water begins to freeze in the extracellular space and then ice crystals dehydrate the cell $[12,13]$. As water begins to freeze in plant, liquid water is converted into solid water which is hard to be used by plants, ultimately causing the available water for plants to decrease sharply. Moreover, freezing stress can induce various damages for plants, including cellular dehydration, structural changes in tissues or organs, embolisms in xylem vessels, frost cracks, and plant diseases [14]. In the process of freezing stress, some protection measures should be adapted against cold damages, such as pruning branches, wrapping the trunk, and painting the trunk with white latex paint.

Disease stress usually occurs after plants are infected with various bacteria, fungus, virus, or insects [15]. Upon infection, the plant's water status would also be affected [16-21]. Roberts and Schreiber investigated the changes of root resistance to water flow in American elm seedlings inoculated with Ceratocystis ulmi and concluded that the water flow of infected plants was reduced to about $40 \%$ as compared with healthy plants [17]. Parke et al. and Collins et al. indicated that tanoak infected with Phytophthora ramorum had significantly more tyloses than noninfected trees in xylem vessels, resulting in the reduction of water transport and hydraulic conductivity $[18,19]$. Park et al. reported that sap flow velocity was significantly lower in bitternut hickory infected with Ceratocystis smalleyi than that in noninfected trees [20]. Ploetz et al. claimed that avocado infected with Raffaelea lauricola had significantly smaller net photosynthetic rate, stomatal conductance, transpiration rate, water use efficiency, and xylem sap flow rate than noninfected trees [21]. In the process of disease stress, plant water status is disturbed, indicating that some preventive measure should be adapted against disease stress.

Based on the above analysis, it can be concluded that plant water status is significantly affected by drought, cold, and disease stress. Therefore, measuring plant water status contributes to a better understanding of these stresses. So far, most researchers use stem sap flow, leaf transpiration rate, and water potential to assess various abiotic and biotic stresses $[10,22]$. But there are few studies about the effects of these stresses on stem water content $(\mathrm{StWC})=($ volume of water):(volume of stem). In this study, we monitored the StWC of crape myrtle trees. Meanwhile, some environmental and physiological parameters were synchronously monitored. The main objectives were to (1) analyze the response of StWC to environmental and physiological parameters, (2) analyze the response of StWC to drought stress, (3) analyze the response of StWC to cold stress, and (4) analyze the response of StWC to disease stress.

\section{Materials and Methods}

2.1. Studying Site. This study was conducted in a nursery $\left(116^{\circ} 20^{\prime} 43.62^{\prime \prime} \mathrm{E}, 40^{\circ} 0^{\prime} 41.92^{\prime \prime} \mathrm{N}\right)$ near Beijing Forestry University. The study area has a temperature monsoon climate with warm summers and cold winters. The main soil type is clay loam with $\mathrm{pH}$ values ranging from 7 to 8 . Since 2015 , the mean annual air temperature was $12.6^{\circ} \mathrm{C}$, and the mean maximum and minimum annual air temperature of the warmest and the coldest months were 36.5 and $-12.8^{\circ} \mathrm{C}$, respectively. The mean annual precipitation was $620 \mathrm{~mm}$ and the mean annual sunshine duration was 2569 hours. A large number of crape myrtle trees were planted in the nursery. These trees with similar morphological characteristics (approx. $2.5 \mathrm{~m}$ in tree height, $4 \mathrm{~cm}$ in diameter at breast height, $1 \mathrm{~m}^{2}$ in crown projection area) were selected as experimental subjects.

\subsection{Measuring Methods}

2.2.1. Measurement of Stem Water Content. StWC was monitored by a self-made high-frequency standing wave ratio (SWR) sensor which had been developed in our previous researches and can detect StWC noninvasively in situ and in real time [23]. Based on the previous researches, the performance indexes of SWR sensor can be concluded as follows: axial sensitive distance $\leq 53 \mathrm{~mm}$, radial sensitive distance $\leq 20 \mathrm{~mm}$, measuring range for StWC $1 \sim 100 \%$, measuring sensitivity $\geq 10 \mathrm{mV} / 1 \%$ and average measuring error $\leq 1 \%$.

\subsubsection{Measurement of Environmental and Physiological} Parameters. Root-water-uptake rate is related with soil temperature (ST) and moisture (SM). Leaf transpiration rate is influenced by air temperature (AT), humidity $(\mathrm{AH})$, and vapor pressure deficit (VPD). Photosynthetic rate is directly determined by photosynthetically active radiation (PAR). Hence, it is necessary to monitor the six environmental parameters which are external factors to affect the regulation of plant water status. Soil temperature and moisture were measured by 5TM sensor (Decagon, America, temperature range $-40 \sim 60^{\circ} \mathrm{C}$, temperature accuracy $\pm 1^{\circ} \mathrm{C}$, moisture range $0 \sim 100 \%$, moisture accuracy $\pm 2 \%$ ). Air temperature and humidity were measured by HMP50-L6 sensor (Vaisala, Finland, temperature range $-10 \sim 50^{\circ} \mathrm{C}$, temperature accuracy $\pm 0.25^{\circ} \mathrm{C}$, humidity range $0 \sim 100 \%$, humidity accuracy $\pm 2 \%$ ). PAR was measured by LI-190R sensor (Li-Cor, America, range $0 \sim 10000 \mu \mathrm{mol} \mathrm{m} \mathrm{m}^{-2} \mathrm{~s}^{-1}$, accuracy $\left.\pm 5 \%\right)$. VPD can be calculated using air temperature and humidity based on the Goff-Gratch formulation [24].

Plant physiological parameters, such as net photosynthetic rate $(\mathrm{Pn})$, transpiration rate $(\mathrm{Tr})$, stomatal conductance (Gs), and intercellular $\mathrm{CO}_{2}$ concentration $(\mathrm{Ci})$ are internal factors to affect the regulation of plant water status. The four physiological parameters were synchronously measured and recorded by LI-6400XT portable photosynthesis system (Li-Cor, America, $\mathrm{CO}_{2}$ range $0 \sim 3100 \mu \mathrm{mol} \mathrm{mol}{ }^{-1}$, $\mathrm{CO}_{2}$ accuracy $\pm 10 \mu \mathrm{mol} \mathrm{mol}^{-1}, \mathrm{H}_{2} \mathrm{O}$ range $0 \sim 75 \mathrm{mmol} \mathrm{mol}^{-1}$, $\mathrm{H}_{2} \mathrm{O}$ accuracy $\left.\pm 1 \mathrm{mmol} \mathrm{mol}^{-1}\right)$. Considering the fact that $\mathrm{Tr}$ measured by LI-6400XT just represents the leaf transpiration 
rate, the whole-tree transpiration was measured by electronic balance (UWA-T-030, LangKe XingYe Weighing Equipment Ltd., China, range $0 \sim 30 \mathrm{~kg}$, accuracy $\pm 1 \mathrm{~g}$ ).

2.3. Experimental Methods. Before conducting the experiments of drought, cold, and disease stress, we monitored StWC of crape myrtle trees under unstressed conditions on a typical day in summer. In this experiment, three well-grown crape myrtle trees were chosen as experimental samples in the nursery. In the meantime, environmental parameters were automatically measured by self-developed data logger in the whole day and plant physiological parameters were manually measured by LI-6400XT portable photosynthesis system from 6:00 to 18:00 at intervals of an hour.

In the experiment of drought stress, crape myrtle trees were divided into experimental and control group with three trees in each group. Each tree was cultivated in a polyresin pot with the volume of $10.2 \mathrm{~L}$. The textural compositions of the soil in the pots were sand $0.61 \mathrm{~g} \mathrm{~g}^{-1}$, silt $0.28 \mathrm{~g} \mathrm{~g}^{-1}$, and clay $0.11 \mathrm{gg}^{-1}$. The soil surface in the experimental group was covered by plastic wrap to prevent water from evaporating. Compared with the experimental trees without irrigation, the trees in the control group were fully irrigated every day. This experiment was conducted in a greenhouse and lasted for ten days between April and May. In the start stage, the drought stress was slight with soil moisture between $9 \%$ and $14 \%$. In the middle stage, the drought stress was moderate with soil moisture between $7 \%$ and $11 \%$. In the end stage, the drought stress was severe with soil moisture between 5\% and 9\%. During the three stages of drought stress, StWC and environmental parameters of all samples were monitored. In addition, the weight of the pot in experimental group was synchronously monitored to calculate the whole-tree transpiration.

In the experiment of cold stress, three crape myrtle trees were chosen as experimental samples in the nursery. In order to observe the responses of StWC to warm and cold weather, respectively, under natural environment, the experiment was divided into two stages. In the first stage, the experiment was carried out in early September when the trees were in growth period and the air temperature ranged from 17.9 to $30.9^{\circ} \mathrm{C}$. In the second stage, the experiment was carried out in late November when the trees were in dormancy period and the air temperature ranged from -5.6 to $7.9^{\circ} \mathrm{C}$. During both stages, StWC and environmental parameters of all samples were monitored.

Based on historical cultivation experience in the nursery, Eriococcus legerstroemiae Kuwana usually parasitize in the cracks of the trunk in winter, thus causing the crape myrtle trees suffering from sooty blotch in June. In order to obtain different disease degree of samples, crape myrtle trees were divided into three groups with 24 trees in each group and disease treatments, such as pruning branches and spraying lime-sulfur mixture, were adopted for the three groups with different frequencies. In the first group, disease treatments were taken both in winter and spring and then the trees were healthy without disease (no black spots on leaves). In the second group, the same disease treatments were taken only in spring and then the trees were slightly infected with sooty blotch (black spot area less than $1 / 3$ of leaf area). In the third group, no disease treatment was taken at any time and then the trees were severely infected with sooty blotch (black spot area more than $1 / 3$ of leaf area). During the germination process of crape myrtle tree, StWC and environmental parameters of all samples were monitored. This experiment was conducted in the nursery from May to July.

2.4. Data Collection and Preprocessing. The data including StWC and environmental parameters were collected using the self-developed data logger (8 MB flash, 16-channel, and 12-bit ADC, 0 2.5 V range, 2-channel RS-232, 2-channel RS-485) at intervals of 10 minutes. The sensors measuring environmental parameters were installed on the selfdesigned bracket (Figure 1(a)) and the SWR sensor measuring StWC was installed on the tree stem at breast height which is set to $1.3 \mathrm{~m}$ above the ground in forestry (Figure 1(b)). Then, all sensors mentioned above were connected to the data logger. In the process of data collection, the sensor data was affected by missing data and abnormal values due to instrumental error or some other external factors like severance of connectivity or power shutdown. These missing data was interpolated using rolling average of available data of past three samples. These abnormal values lying outside the permissible range for the corresponding parameter were also replaced by rolling average of past three samples. Then, all data was further denoised using mean filtering with the window length of three.

\section{Results and Discussion}

3.1. Response of StWC to Environmental and Physiological Parameters. Drought and cold stress are essentially determined by environmental parameters. Then, these stresses in turn will affect plant physiological parameters. Therefore, it is necessary to analyze the response of StWC to environmental and physiological parameters under natural environment before analyzing other experiments including drought and cold stress.

The correlations between StWC and environmental parameters were analyzed under unstressed conditions on a typical day in summer (Table 1). Correlation analysis suggests that AT, PAR, and VPD were significantly negatively correlated with StWC and AH was significantly positively correlated with StWC. Considering that AT and AH can be characterized by VPD, we further analyzed the diurnal variation rule between StWC and environmental parameters including PAR and VPD. As can be seen from Figure 2, when PAR or VPD increased in the morning, water loss rate by leaf transpiration was greater than water absorption rate by root, thus resulting in the fall of StWC. Nevertheless, as PAR or VPD reached a certain threshold at midday, there was a dynamic balance between water loss and water absorption due to the midday depression, thus resulting in small fluctuations of StWC. When PAR or VPD decreased in the afternoon, water loss rate by leaf transpiration was smaller than water absorption rate by root, thus resulting in the raise of StWC $[25,26]$. 


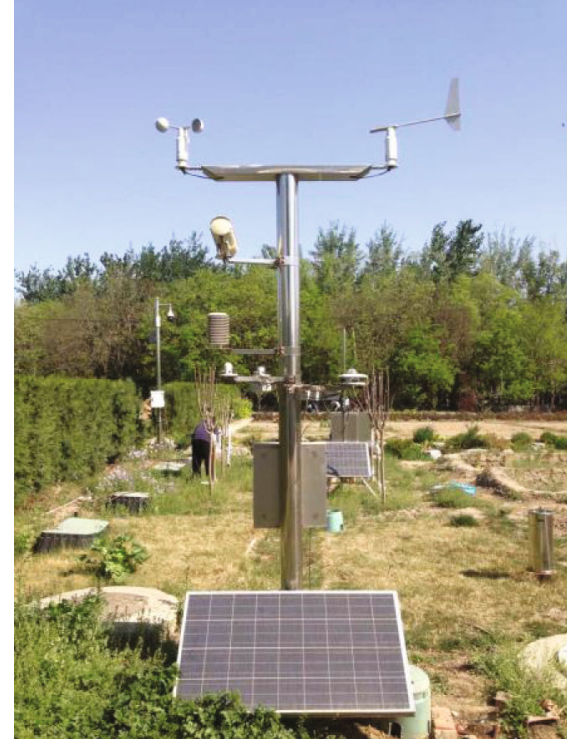

(a)

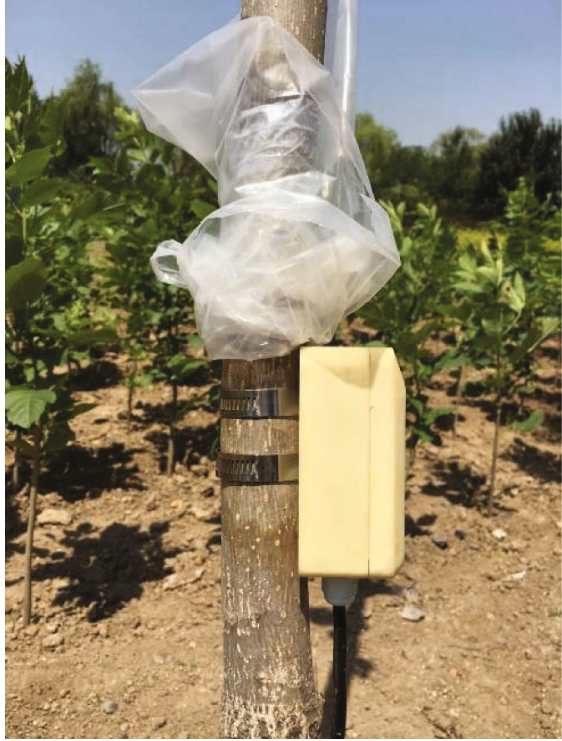

(b)

FIGURE 1: (a) The installation of environmental sensors. (b) The installation of SWR sensor.

TABLE 1: Correlations between StWC and environmental parameters under unstressed conditions on a typical day in summer.

\begin{tabular}{lccccccc}
\hline \multirow{2}{*}{ Variables } & & \multicolumn{2}{c}{ Environmental parameters } \\
& & ST & SM & AT & AH & PAR & VPD \\
\hline \multirow{3}{*}{ StWC } & Correlation coefficient & -0.0284 & -0.1873 & -0.5875 & 0.5372 & -0.9132 \\
& Significance probability & 0.8954 & 0.3809 & 0.0025 & 0.0068 & 0.0000 \\
& Sample size & 24 & 24 & 24 & 24 & 24 \\
\hline
\end{tabular}

ST: soil temperature; SM: soil moisture; AT: air temperature; AH: air humidity; PAR: photosynthetically active radiation; VPD: vapor pressure deficit.

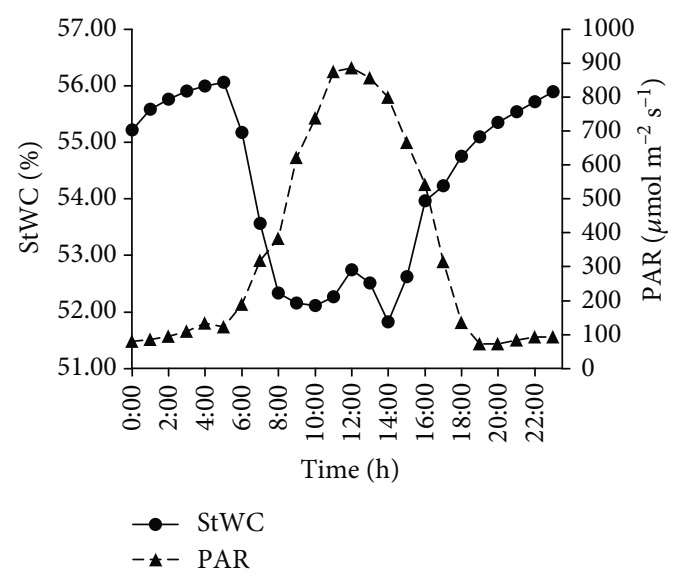

(a)

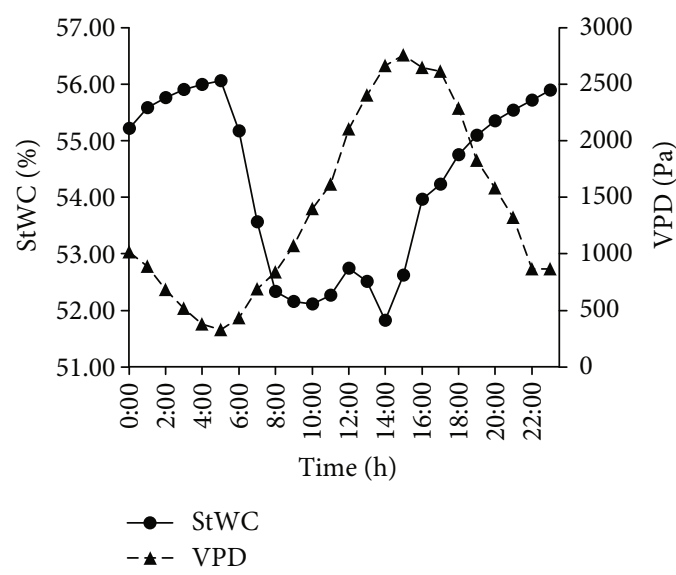

(b)

FIGURE 2: Diurnal variation rule between StWC and environmental parameters including PAR (a) and VPD (b) under unstressed conditions on a typical day in summer.

The correlations between StWC and physiological parameters were synchronously analyzed in the same case (Table 2). Correlation analysis suggests that $\mathrm{Pn}, \mathrm{Tr}$, and Gs were significantly negatively correlated with StWC and Ci was significantly positively correlated with StWC. Comparing Table 1 with Table 2, it can be concluded that the 
TABLE 2: Correlations between StWC and physiological parameters under unstressed conditions on a typical day in summer.

\begin{tabular}{|c|c|c|c|c|c|}
\hline \multirow{2}{*}{ Variables } & & \multicolumn{4}{|c|}{ Physiological parameters } \\
\hline & & Pn & $\operatorname{Tr}$ & Gs & $\mathrm{Ci}$ \\
\hline \multirow{3}{*}{ StWC } & Correlation coefficient & -0.8402 & -0.8374 & -0.8535 & 0.9457 \\
\hline & Significance probability & 0.0003 & 0.0004 & 0.0002 & 0.0000 \\
\hline & Sample size & 13 & 13 & 13 & 13 \\
\hline
\end{tabular}

Pn: net photosynthetic rate; Tr: transpiration rate; Gs: stomatal conductance; Ci: intercellular $\mathrm{CO}_{2}$ concentration.

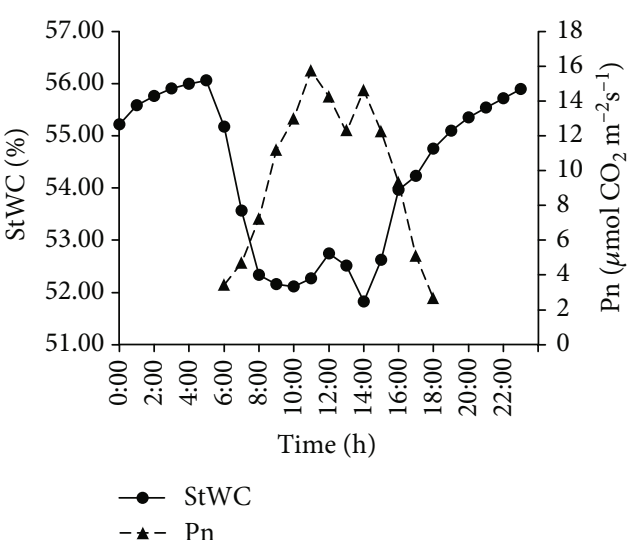

(a)

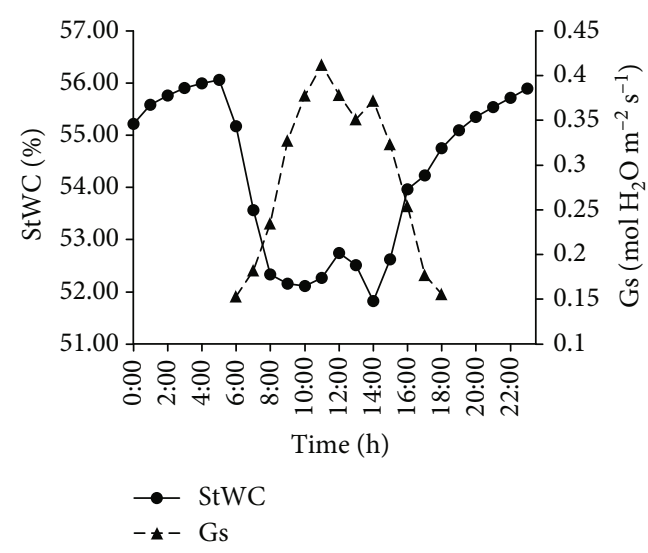

(c)

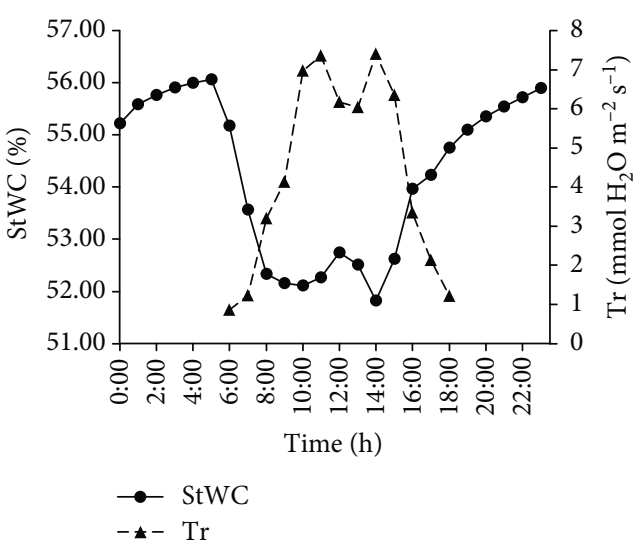

(b)

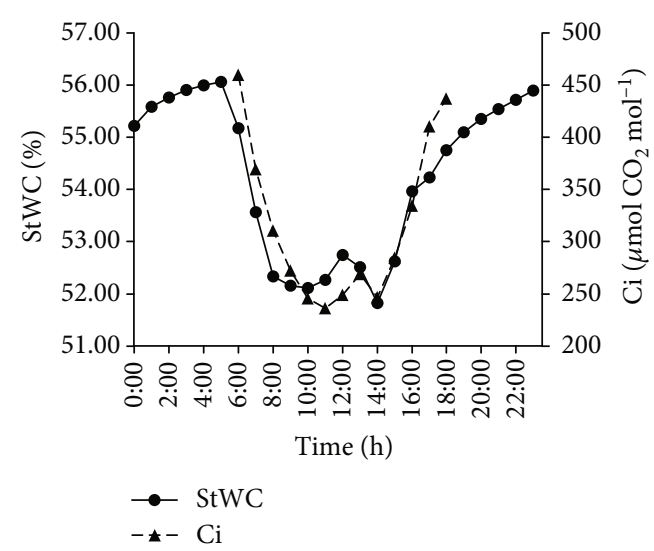

(d)

Figure 3: Diurnal variation rule between StWC and environmental parameters including $\operatorname{Pn}(\mathrm{a}), \operatorname{Tr}(\mathrm{b})$, Gs (c), and Ci (d) under unstressed conditions on a typical day in summer.

correlation coefficients between StWC and physiological parameters were generally greater than that between StWC and environmental parameters. The reason for the difference may be that the physiological parameters directly affect StWC, but the environmental parameters indirectly affect StWC by changing the physiological parameters [27, 28]. In addition, we further analyzed the diurnal variation rule between StWC and the four physiological parameters (Figure 3). Comparing Figure 2 with Figure 3, the diurnal variation rule between StWC and physiological parameters excluding $\mathrm{Ci}$ was similar with that between StWC and environmental parameters. And there was an obvious midday depression of $\mathrm{Pn}$ and Gs, thus resulting in the rise of $\mathrm{Ci}$ and the fall of $\mathrm{Tr}$, respectively [29, 30]. Therefore, the StWC also increased at midday. The $\mathrm{Tr}$ decreased from 11:00 to 13:00, during which time water loss rate by leaf transpiration was smaller than water absorption rate by root, thus resulting in the rise of StWC from 10:00 to 12:00. The $\mathrm{Tr}$ increased from 13:00 to 14:00, during which time water loss rate by leaf transpiration was greater than water absorption rate by root, thus resulting in the fall of StWC from 12:00 to 14:00. And the change of StWC was ahead of the change of $\operatorname{Tr}$ about an hour. Based on the above analysis, the conclusion can be drawn that StWC 


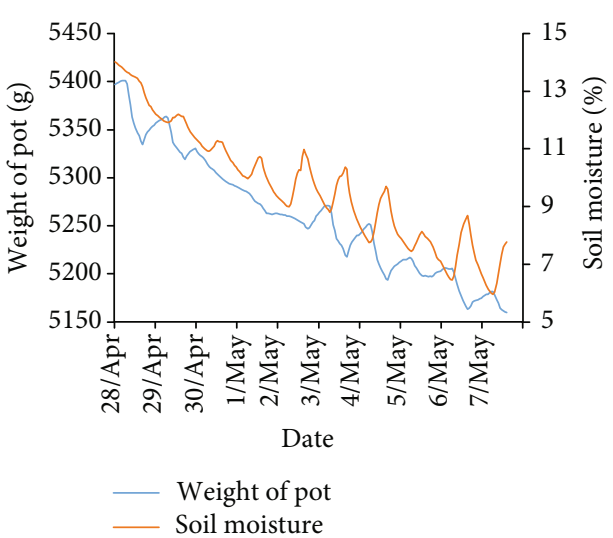

(a)

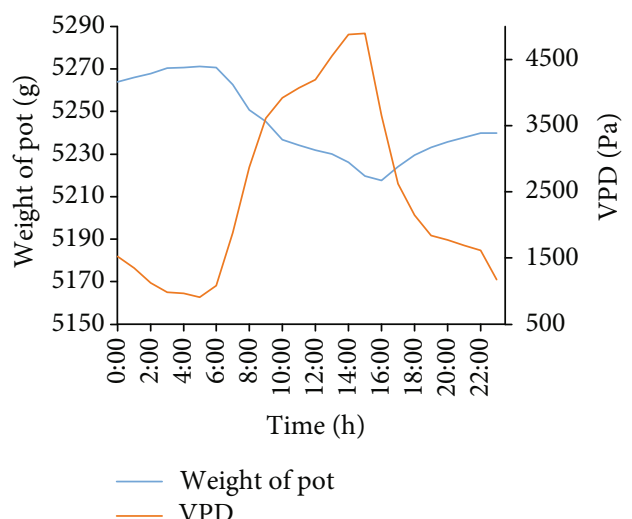

(c)

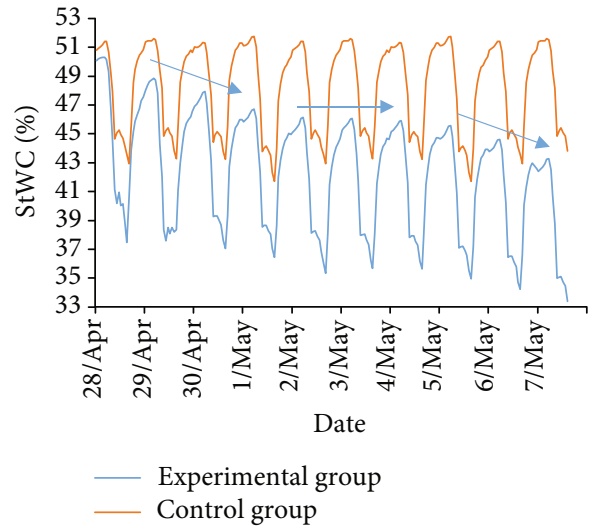

(b)

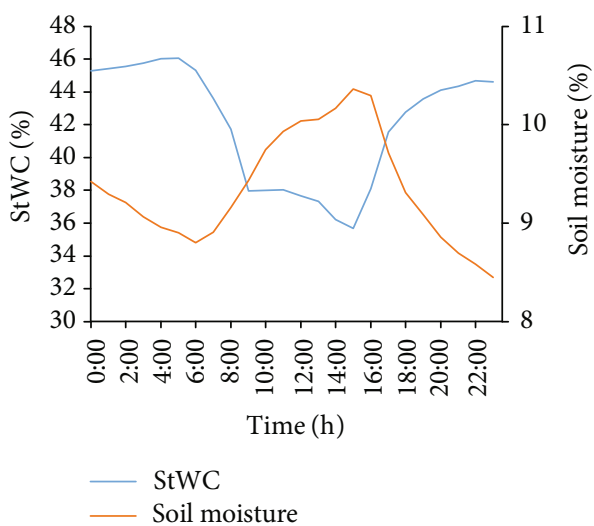

(d)

FIgUre 4: Response of StWC to drought stress. (a) Periodic variation rule between weight of pot and soil moisture in the experimental group. (b) Periodic variation rule of StWC in the experimental and control groups. (c) Diurnal variation rule between weight of pot and VPD in the experimental group on May 3. (d) Diurnal variation rule between StWC and soil moisture in the experimental group on May 3.

can characterize the environmental and physiological information to some extent.

3.2. Response of StWC to Drought Stress. The response of StWC to drought stress is shown in Figure 4. From an overall perspective, the weight of pot and soil moisture continuously declined throughout the period of drought stress (Figure 4(a)). As shown in Figure 4(b), the diurnal mean StWC of the tree in the control group with adequate irrigation was steady and the diurnal mean StWC of the tree in the experimental group without irrigation showed diversity which can be divided into three stages. In the start stage of drought stress (from April 28 to May 1), the water potential of the tree was higher than that of the soil and then the water content of the tree would decrease, resulting in the reduction of the diurnal mean StWC. In the middle stage of drought stress (from May 2 to May 4), as the drought stress became severe (soil moisture less than $11 \%$ ), the water potential of the tree would equilibrate with that of the soil by accumulation of solutes and cell wall hardening [10], resulting in the stabilization of the diurnal mean StWC (approx. 42\%). The length of stable time was dependent on the drought resistance of crape myrtle. In the end stage of drought stress (from
May 5 to May 7), as the drought stress became more severe (soil moisture less than 9\%), the balance of water potential between tree and soil was destroyed, resulting the further decline of the diurnal mean StWC. When plants are in the end stage of drought stress, some morphological and biochemical responses of plants will cause negative effects on plants [31]. In terms of morphological response, drought stress can cause impaired mitosis, cell elongation, and expansion resulting in reduced growth and yield traits $[32,33]$. In terms of biochemical response, drought stress can cause the increase of ROS level resulting in oxidative damage to proteins, DNA, and lipids [9]. Hence, in order to reduce the damages for plants, the diurnal mean StWC in the middle stage of drought stress can be regarded as the critical threshold of irrigation.

In this experiment, some interesting phenomena were observed. As shown in Figure 4(a), the weight of pot showed a downward fluctuation trend. Compared with the period from April 30 to May 2, the weight of pot changed more significantly between May 3 and May 4. As shown in Figure 4(c), the weight of the pot appeared to have an obvious increase (approx. $29 \mathrm{~g}$ ) at night. This phenomenon may be caused by two factors. One factor was that the VPD is usually 

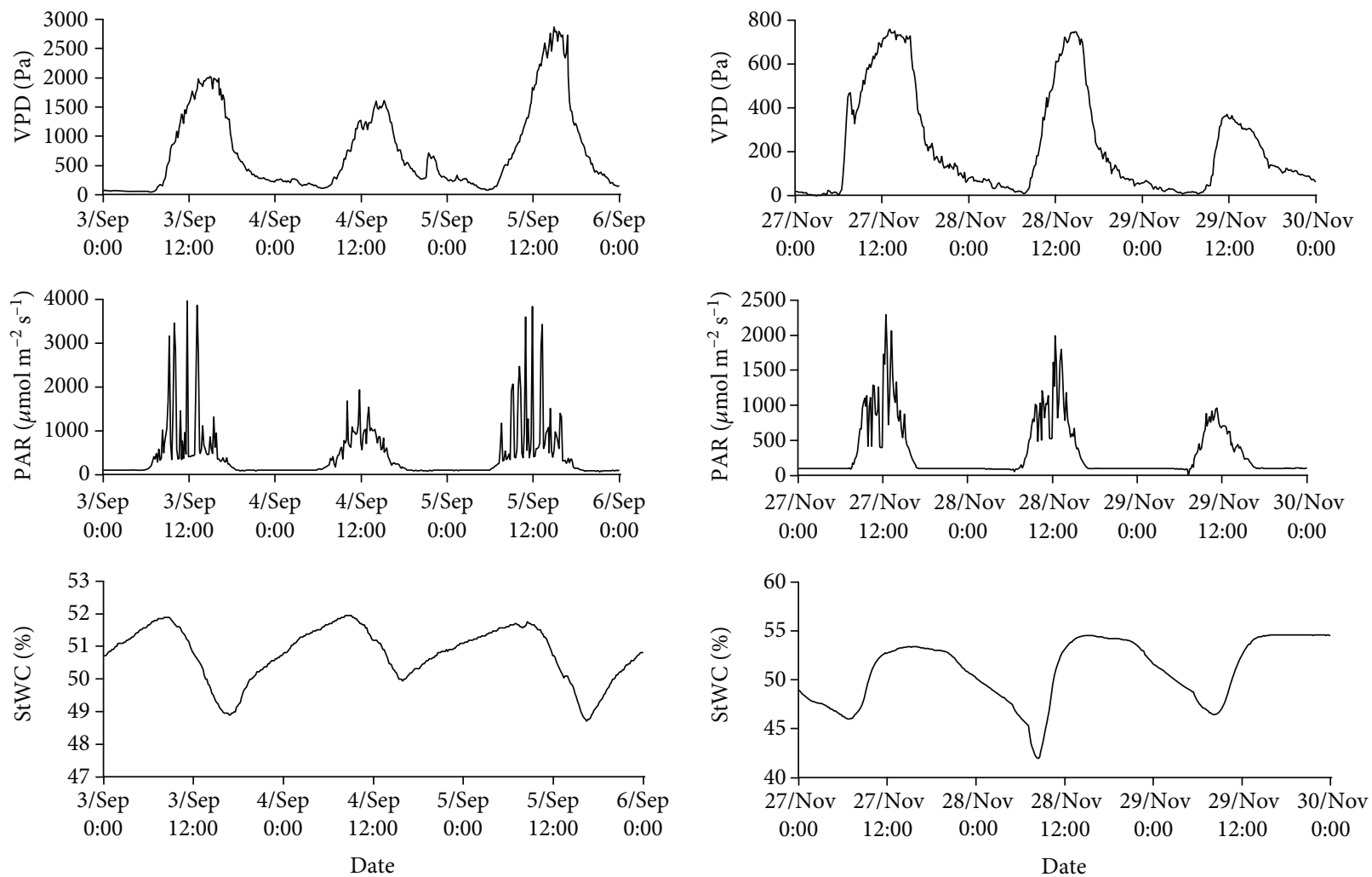

(a)

(b)

Figure 5: Responses of VPD, PAR, and StWC to temperature stress. (a) Periodic variation rules of VPD, PAR, and StWC in warm weather. (b) Periodic variation rules of VPD, PAR, and StWC in cold weather.

very low in the greenhouse at night resulting in the formation of condensation water on leaves [34]. The other factor was that the transpiration of plants is usually extremely weak at night and the growth rate of plants at night is faster than that in the daytime resulting in the accumulation of biomass [35]. Meanwhile, as shown in Figure 4(d), the soil moisture in pot appeared to have a slight increase (approx. 1.6\%) during the daytime. This phenomenon may be caused by strong transpiration resulting in the increase of soil moisture near the roots where the 5TM sensor was placed [36].

3.3. Response of StWC to Cold Stress. The responses of VPD, $\mathrm{PAR}$, and StWC to temperature stress are shown in Figure 5. As shown in Figure 5(a), in warm weather with high VPD and PAR, StWC gradually increased at night and decreased during the daytime. As shown in Figure 5(b), in cold weather with low VPD and PAR, StWC gradually decreased at night and increased during the daytime. Therefore, it can be concluded that the StWC showed opposite diurnal variation rules in warm and cold weather, respectively. In addition, the mean diurnal range of StWC in cold weather (approx. 9.4\%) was significantly larger than that in warm weather (approx. 2.7\%). In order to further analyze the response of StWC to cold stress, the periodic variation rule between StWC and air temperature in cold weather is shown in Figure 6 . When the air temperature fell below $0^{\circ} \mathrm{C}$ at night, the StWC slowly decreased. When the air temperature rose

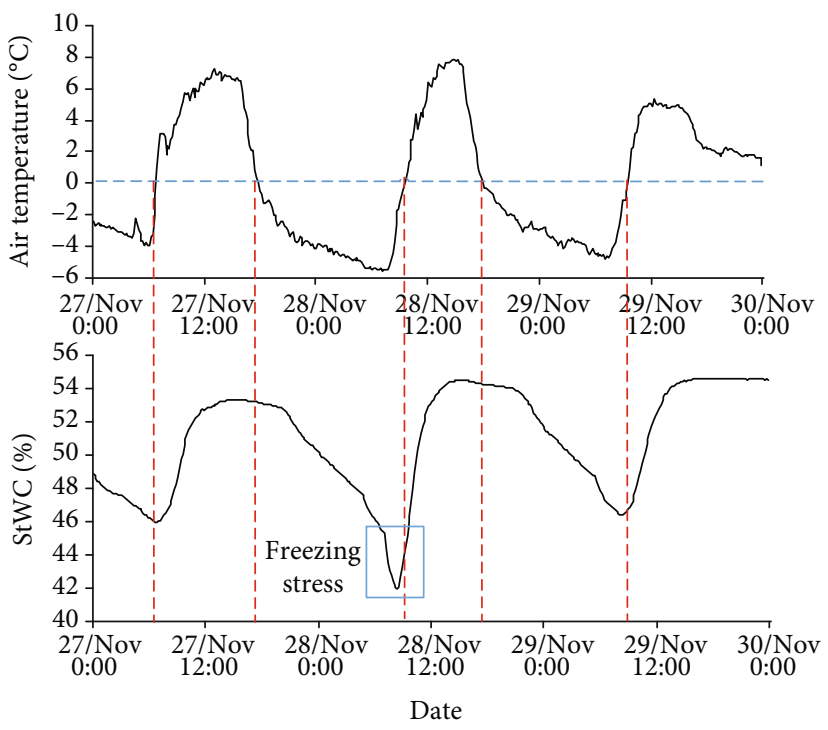

Figure 6: Periodic variation rule between StWC and air temperature in cold weather.

above $0^{\circ} \mathrm{C}$ during the daytime, the StWC gradually increased. Considering that chilling stress can restrict root water uptake by decreasing the VPD [37] and increasing the viscosity of soil moisture [38], then it caused the slow decrease of the 
StWC. Hence, the conclusion can be drawn that the plant may suffer from chilling stress when the air temperature is lower than $0^{\circ} \mathrm{C}$. When the air temperature fell below $-5^{\circ} \mathrm{C}$ on November 28 , the StWC sharply decrease by $5.5 \%$ in 210 minutes. When the air temperature rose above $-5^{\circ} \mathrm{C}$ on November 28, the StWC sharply increased by $9.8 \%$ in 160 minutes. Considering that freezing stress can induce the formation of ice in the large vessels of the xylem in stems [11], then it caused the sharp decrease of the StWC. Therefore, it can be concluded that the plant may suffer from freezing stress when the air temperature is lower than $-5^{\circ} \mathrm{C}$. Based on the above analysis, we can conclude that cold stress can weaken the water regulation ability of plants and then result in the larger diurnal fluctuation of StWC.

3.4. Response of StWC to Disease Stress. The StWC changing curves of crape myrtle with different health levels during the period of germination are shown in Figure 7. In the long term, the diurnal mean StWC of healthy and mild disease tree decreased firstly then increased. However, the diurnal mean StWC of severe disease tree decreased all the time. Considering that the better understanding of the effects of disease on StWC can contribute to the early diagnosis of the disease, we further analyzed the diurnal variation rule of StWC under disease stress. The diurnal minimum value, maximum value, mean value, and range value of StWC were selected as feature parameters which were used to represent the diurnal variation rule of StWC. The means and standard deviations of the four feature parameters among different health level groups on June 1 are shown in Figure 8. The health level of the groups, in descending order, was healthy group $>$ mild disease group $>$ severe disease group. The diurnal minimum value, maximum value and mean value of StWC showed a positive correlation with the health level of the group and the diurnal range value of StWC showed a negative correlation with the health level of the group. Meanwhile, the effects of disease on the four feature parameters were tested using one-way ANOVA analysis. As can be seen from Table 3, the four feature parameters differed significantly $(p<0.001)$ among different health level groups, indicating that the health status of the tree can be diagnosed by analyzing the four feature parameters. The effects of disease on StWC also can be interpreted based on plant physiology. The cankers caused by disease can induce the formation of tyloses which can cause the reduced hydraulic conductivity, xylem function [39], and water transport [40] in affected stems, ultimately resulting in the decrease of diurnal mean value of StWC and the increase of diurnal range value of StWC.

\section{Conclusions}

In this study, the novel SWR sensor was used to monitor the StWC of crape myrtle trees. The responses of StWC to environmental and physiological parameters, drought, cold, and disease stress were analyzed, respectively. In the meantime, the effects of the three types of stress on StWC were interpreted based on related mechanisms. The results proved that the StWC was simultaneously affected by both environmen-

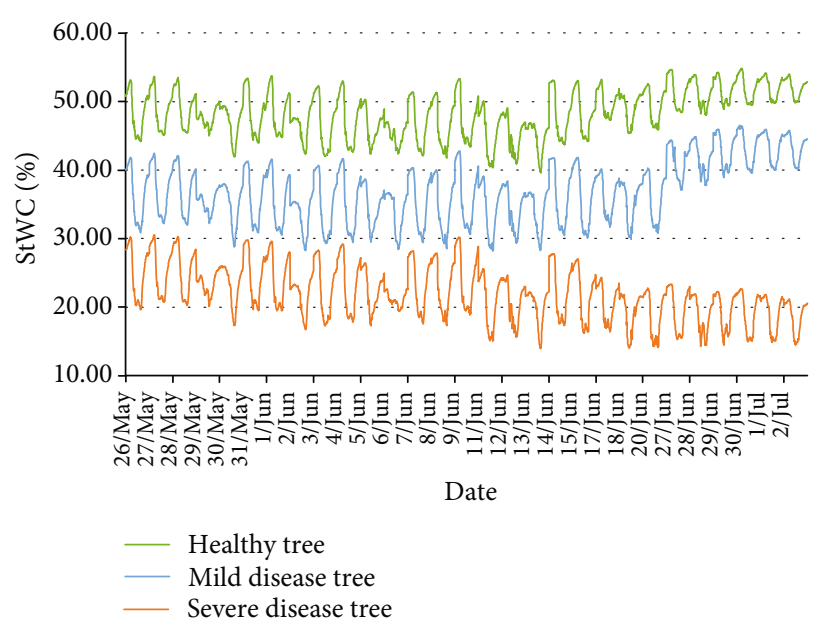

FIGURE 7: StWC changing curves of crape myrtle with different health levels during the period of germination.

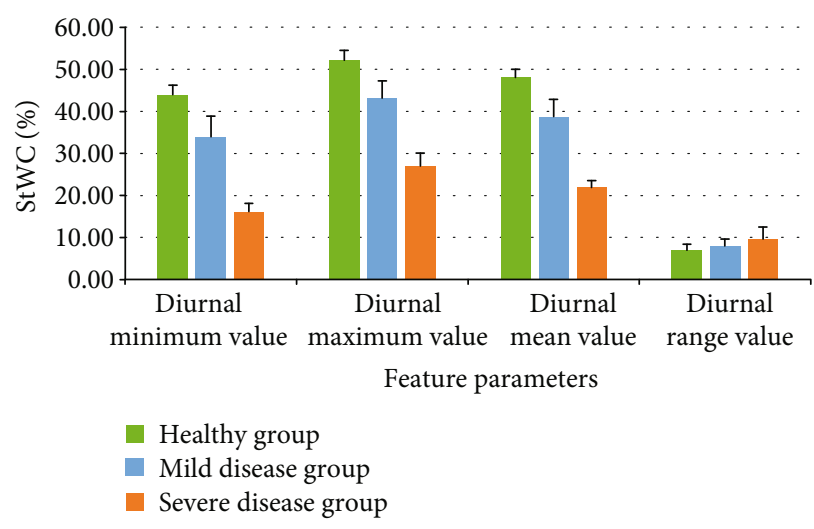

FIgURE 8: The means and standard deviations of four feature parameters among different groups on June 1.

tal parameters determined by stress degree and physiological parameters. To some extent, both were coupled with each other and difficult to separate. Therefore, it was difficult to quantitatively evaluate the effects of drought, cold, and disease stress on StWC. But the StWC still can be used as a qualitative evaluation index of the degree of the three types of stress.

(1) In the start and end stage of drought stress, there was a negative correlation between the diurnal mean of StWC and the degree of drought stress. In the middle stage, the diurnal mean of StWC remained stable for a period of time; the length of which can characterize the drought resistance of plants

(2) In the case of cold stress, the StWC showed opposite diurnal variation rules in warm and cold weather, respectively. There was a positive correlation between the diurnal range of StWC and the degree of cold stress. The decline rate of StWC under chilling stress was significantly less than that under freezing stress 
TABLE 3: Tests for disease effects on four feature parameters using one-way ANOVA analysis.

\begin{tabular}{|c|c|c|c|c|c|c|}
\hline Feature parameters & Source & Sum of squares & Df & Mean square & $F$ & Sig. \\
\hline \multirow{2}{*}{ Diurnal minimum StWC } & Between groups & 0.953 & 2 & 0.476 & 421.562 & $<0.001$ \\
\hline & Within groups & 0.078 & 69 & 0.001 & & \\
\hline \multirow{2}{*}{ Diurnal maximum StWC } & Between groups & 0.778 & 2 & 0.389 & 368.243 & $<0.001$ \\
\hline & Within groups & 0.073 & 69 & 0.001 & & \\
\hline \multirow{2}{*}{ Diurnal mean StWC } & Between groups & 0.842 & 2 & 0.421 & 534.468 & $<0.001$ \\
\hline & Within groups & 0.054 & 69 & 0.001 & & \\
\hline \multirow{2}{*}{ Diurnal range StWC } & Between groups & 0.009 & 2 & 0.004 & 10.350 & $<0.001$ \\
\hline & Within groups & 0.029 & 69 & $<0.001$ & & \\
\hline
\end{tabular}

(3) In the case of disease stress, the diurnal minimum, maximum, and mean of StWC showed a positive correlation with the health level of plants and the diurnal range of StWC showed a negative correlation with the health level of plants

\section{Data Availability}

The data used to support the findings of this study are available from the corresponding author upon request.

\section{Conflicts of Interest}

The authors declare that they have no conflicts of interest.

\section{Authors' Contributions}

For this research article, Chao Gao and Yandong Zhao conceived and designed the experiments. Chao Gao performed the experiments. Chao Gao and Yue Zhao analyzed the data. Chao Gao, Yue Zhao, and Yandong Zhao wrote the paper.

\section{Acknowledgments}

This research was funded by the Research Foundation for Youth Scholars of Beijing Technology and Business University (Grant No. QNJJ2020-23), the National Key Research and Development Program of China (Grant No. 2017YFD0600901), the Beijing Municipal Science and Technology Commission (Grant No. Z161100000916012), and the Special Fund for Beijing Common Construction Project.

\section{References}

[1] C. A. Jaleel, P. Manivannan, A. Wahid et al., "Drought stress in plants: a review on morphological characteristics and pigments composition," International Journal of Agriculture and Biology, vol. 11, no. 1, pp. 100-105, 2009.

[2] R. M. Augé, "Water relations, drought and vesiculararbuscular mycorrhizal symbiosis," Mycorrhiza, vol. 11, no. 1, pp. 3-43, 2001.

[3] J. S. Sperry, Y. Wang, B. T. Wolfe et al., "Pragmatic hydraulic theory predicts stomatal responses to climatic water deficits," New Phytologist, vol. 212, no. 3, pp. 577-589, 2016.
[4] J. Zhang, H. T. Nguyen, and A. Blum, "Genetic analysis of osmotic adjustment in crop plants," Journal of Experimental Botany, vol. 50, no. 332, pp. 291-302, 1999.

[5] M. P. Apse and E. Blumwald, "Engineering salt tolerance in plants," Current Opinion in Biotechnology, vol. 13, no. 2, pp. 146-150, 2002.

[6] R. Murphy and J. K. E. Ortega, "A new pressure probe method to determine the average volumetric elastic modulus of cells in plant tissue," Plant Physiology, vol. 107, no. 3, pp. 995-1005, 1995.

[7] T. J. Close, "Dehydrins: a commonalty in the response of plants to dehydration and low temperature," Physiologia Plantarum, vol. 100, no. 2, pp. 291-296, 1997.

[8] D. K. Hincha and M. Hagemann, "Stabilization of model membranes during drying by compatible solutes involved in the stress tolerance of plants and microorganisms," Biochemical Journal, vol. 383, no. 2, pp. 277-283, 2004.

[9] K. Apel and H. Hirt, "Reactive oxygen species: metabolism, oxidative stress, and signal transduction," Annual Review of Plant Biology, vol. 55, pp. 373-399, 2004.

[10] P. E. Verslues, M. Agarwal, S. Katiyar-Agarwal, J. Zhu, and J. K. Zhu, "Methods and concepts in quantifying resistance to drought, salt and freezing, abiotic stresses that affect plant water status," The Plant Journal, vol. 45, no. 4, pp. 523-539, 2006.

[11] N. S. Arias, F. G. Scholz, G. Goldstein, and S. J. Bucci, "The cost of avoiding freezing in stems: trade-off between xylem resistance to cavitation and supercooling capacity in woody plants," Tree Physiology, vol. 37, no. 9, pp. 1251-1262, 2017.

[12] R. S. Pearce, "Extracellular ice and cell shape in frost-stressed cereal leaves: a low-temperature scanning-electron-microscopy study," Planta, vol. 175, no. 3, pp. 313-324, 1988.

[13] R. S. Pearce and E. N. Ashworth, "Cell shape and localisation of ice in leaves of overwintering wheat during frost stress in the field," Planta, vol. 188, no. 3, pp. 324-331, 1992.

[14] R. S. Pearce, "Plant freezing and damage," Annals of Botany, vol. 87, no. 4, pp. 417-424, 2001.

[15] S. Sankaran, A. Mishra, R. Ehsani, and C. Davis, "A review of advanced techniques for detecting plant diseases," Computers and Electronics in Agriculture, vol. 72, no. 1, pp. 1-13, 2010.

[16] J. Urban and M. Dvořák, "Sap flow-based quantitative indication of progression of Dutch elm disease after inoculation with Ophiostoma novo-ulmi," Trees, vol. 28, no. 6, pp. 15991605, 2014. 
[17] B. R. Roberts and L. R. Schreiber, "Influence of Dutch elm disease on resistance to water flow through roots of American elm," Phytopathology, vol. 77, no. 1, pp. 56-59, 1977.

[18] J. L. Parke, E. Oh, S. Voelker, E. M. Hansen, G. Buckles, and B. Lachenbruch, "Phytophthora ramorum colonizes tanoak xylem and is associated with reduced stem water transport," Phytopathology, vol. 97, no. 12, pp. 1558-1567, 2007.

[19] B. R. Collins, J. L. Parke, B. Lachenbruch, and E. M. Hansen, "The effects of Phytophthora ramorum infection on hydraulic conductivity and tylosis formation in tanoak sapwood," Canadian Journal of Forest Research, vol. 39, no. 9, pp. 17661776, 2009.

[20] J. H. Park, J. Juzwik, and J. Cavender-Bares, "Multiple Ceratocystis smalleyi infections associated with reduced stem water transport in bitternut hickory," Phytopathology, vol. 103, no. 6, pp. 565-574, 2013.

[21] R. C. Ploetz, B. Schaffer, A. I. Vargas, J. L. Konkol, J. Salvatierra, and R. Wideman, "Impact of laurel wilt, caused by Raffaelea lauricola, on leaf gas exchange and xylem sap flow in avocado, Persea americana," Phytopathology, vol. 105, no. 4, pp. 433-440, 2015.

[22] A. Stöhr and R. Lösch, "Xylem sap flow and drought stress of Fraxinus excelsior saplings," Tree Physiology, vol. 24, no. 2, pp. 169-180, 2004.

[23] C. Gao, Y. Zhao, and Y. Zhao, "A Novel Sensor for Noninvasive Detection of In Situ Stem Water Content Based on Standing Wave Ratio," Journal of Sensors, vol. 2019, Article ID 3594964, 10 pages, 2019.

[24] J. A. Goff, "Saturation pressure of water on the new kelvin temperature scale," in Proc. Semi-annual Meeting of the American Society of Heating and Ventilating Engineers, pp. 347-354, American Society of Heating and Ventilating Engineers, Murray Bay, QC, Canada, 1957.

[25] P. J. Kramer, "The relation between rate of transpiration and rate of absorption of water in plants," American Journal of Botany, vol. 24, no. 1, pp. 10-15, 1937.

[26] M. J. Aston and D. W. Lawlor, "The relationship between transpiration, root water uptake, and leaf water potential," Journal of Experimental Botany, vol. 30, no. 1, pp. 169$181,1979$.

[27] D. A. Grantz, D. Zinsmeister, and J. Burkhardt, "Ambient aerosol increases minimum leaf conductance and alters the aperture-flux relationship as stomata respond to vapor pressure deficit (VPD)," New Phytologist, vol. 219, no. 1, pp. 275286, 2018.

[28] R. Terada, Y. Watanabe, M. Fujimoto, I. Tatamidani, S. Kokubu, and G. N. Nishihara, "The effect of PAR and temperature on the photosynthetic performance of a freshwater red alga, Thorea gaudichaudii (Thoreales) from Kagoshima, Japan," Journal of Applied Phycology, vol. 28, no. 2, pp. 12551263, 2016.

[29] G. Ren, X. Liu, and Y. Shi, "Effects of Plant Growth Regulator S-Y on Diurnal Changes in Photosynthetic Parameters and Yield of Stevia Rebaudina Bertoni," Energy Procedia, vol. 5, pp. 429-434, 2011.

[30] L. V. Chengguo, M. Lei, and S. Yan, "Study on the diurnal changes of net photosynthetic rate and the impact factors of Stevia rebaudiana Bertoni in autumn," American Journal of Plant Physiology, vol. 4, no. 1, pp. 18-23, 2009.

[31] S. A. Anjum, X. Y. Xie, L. C. Wang, M. F. Saleem, C. Man, and W. Lei, "Morphological, physiological and biochemical responses of plants to drought stress," African Journal of Agricultural Research, vol. 6, no. 9, pp. 2026-2032, 2011.

[32] H. Nonami, "Plant water relations and control of cell elongation at low water potentials," Journal of Plant Research, vol. 111, no. 3, pp. 373-382, 1998.

[33] M. Hussain, M. A. Malik, M. Farooq, M. Y. Ashraf, and M. A. Cheema, "Improving drought tolerance by exogenous application of glycinebetaine and salicylic acid in sunflower," Journal of Agronomy and Crop Science, vol. 194, no. 3, pp. 193-199, 2008.

[34] D. Piscia, J. I. Montero, E. Baeza, and B. J. Bailey, "A CFD greenhouse night-time condensation model," Biosystems Engineering, vol. 111, no. 2, pp. 141-154, 2012.

[35] A. Graf, A. Schlereth, M. Stitt, and A. M. Smith, "Circadian control of carbohydrate availability for growth in Arabidopsis plants at night," Proceedings of the National Academy of Sciences, vol. 107, no. 20, pp. 9458-9463, 2010.

[36] H. J. Tromp-van Meerveld and J. J. McDonnell, “On the interrelations between topography, soil depth, soil moisture, transpiration rates and species distribution at the hillslope scale," Advances in Water Resources, vol. 29, no. 2, pp. 293-310, 2006.

[37] R. Aroca, "Involvement of abscisic acid in leaf and root of maize (Zea mays L.) in avoiding chilling-induced water stress," Plant Science, vol. 165, no. 3, pp. 671-679, 2003.

[38] A. J. Bloom, M. A. Zwieniecki, J. B. Passioura, L. B. Randall, N. M. Holbrook, and D. A. St Clair, "Water relations under root chilling in a sensitive and tolerant tomato species," Plant, Cell and Environment, vol. 27, no. 8, pp. 971-979, 2004.

[39] S. A. Inch and R. C. Ploetz, "Impact of laurel wilt, caused by Raffaelea lauricola, on xylem function in avocado, Persea americana," Forest Pathology, vol. 42, no. 3, pp. 239-245, 2012.

[40] T. Kirisits and I. Offenthaler, "Xylem sap flow of Norway spruce after inoculation with the blue-stain fungus Ceratocystis polonica," Plant Pathology, vol. 51, no. 3, pp. 359-364, 2002. 